\title{
Syndrome De Guillain-Barré Chez L'enfant Dans Un Contexte A Ressources Limitées : A Propos De Deux Observations Au CHU Sylvanus Olympio De Lomé - TOGO
}

\author{
Dae. Akolly \\ Km. Guedenon \\ E. Tsolenyanu \\ E. Takassi \\ M. Fiawoo \\ Y. Atakouma
}

Service de Pédiatrie CHU Sylvanus Olympio de Lomé (TOGO).

doi: 10.19044/esj.2016.v12n36p182 URL:http://dx.doi.org/10.19044/esj.2016.v12n36p182

\begin{abstract}
Guillain-Barré syndrome is rare in children. With polyvalent immunoglobulins the management and prognosis has been significantly improved with complete recovery rate exceeding $80 \%$. We report the cases of two children who completely recovered after Guillain-Barré syndrome in the absence of immune globulin. He was a 12 year old daughter and an infant of 17 months male who presented a progressive ascending motor deficit, dysphonia with difficulty swallowing, difficulty breathing, areflexia. A albumino-cytological dissociation was found in the study of cerebrospinal fluid in both cases. Electromyography in the girl had confirmed a axono-form myelin. Retroviral serology was negative in both cases. Other serology (Campylobacter jejuni, Cytomegalovirus, Epstein Barr, ZIKA), the dosage of anti-ganglioside antibodies and brain imaging had not been made. The immunoglobulins are not available in our work context, we conducted a symptomatic management. Complete healing was achieved with a 4-month decline.
\end{abstract}

Keywords : Guillain-Barré, Child, Togo.

\section{Resume}

Le syndrome de Guillain-Barré est rare chez l'enfant. Grâce aux immunoglobulines polyvalentes la prise en charge et le pronostic ont été sensiblement améliorés avec des taux de récupération complète dépassant les 
$80 \%$. Nous rapportons les cas de deux enfants ayant totalement récupérés après avoir présenté un syndrome de Guillain-Barré en l'absence d'administration d'immunoglobulines. Il s'agissait d'une fille de 12 ans et d'un nourrisson de 17 mois de sexe masculin ayant présenté un déficit moteur ascendant progressif, une dysphonie avec des troubles de la déglutition, des difficultés respiratoires, une aréflexie. Une dissociation albumino-cytologique avait été retrouvée à l'étude du liquide céphalorachidien dans les deux cas. L'électromyogramme chez la fille avait confirmé une forme axonomyélinique. Les sérologies retrovirales étaient négatives dans les deux cas. Les autres sérologies (campylobacter jejuni, cytomégalovirus, Epstein Barr, ZIKA), le dosage des anticorps antigangliosides et l'imagerie cérébrale n'avaient pas été réalisés. Les immunoglobulines polyvalentes n'étant pas disponible dans notre contexte de travail, nous avons procédé à une prise en charge symptomatique. La guérison complète a été obtenue avec un recul de 4 mois.

Mots clés : Guillain-Barré, enfant, Togo.

\section{Introduction}

Le syndrome de Guillain-Barré (SGB) est une neuropathie inflammatoire aigüe du système nerveux périphérique. Les premiers cas ont été rapportés par des neurologues français en 1916 (Guillain G et al, 1916). Son incidence mondiale varie entre 0,6 et 4 cas par an pour 100000 habitants (Hugues $\mathrm{R}$ et al, 2005). Cette affection est rare en pédiatrie et son incidence augmente avec l'âge (Kuwabara S, 2004). Le pronostic est meilleur en comparaison aux formes de l'adulte ( $\mathrm{Wu} \mathrm{X}$ et al, 2016). On distingue quatre formes: la polyradiculonévrite aigüe inflammatoire démyélinisante, la neuropathie aigüe motrice axonale pure, la neuropathie aigüe axonale sensitivomotrice et le syndrome de Miller Fisher. L'atteinte respiratoire et dysautonomique constitue le principal facteur de mauvais pronostic pouvant conduire au décès. Les taux de létalité dans les différentes séries pédiatriques sont faibles. Le traitement repose sur l'utilisation des immunoglobulines polyvalentes en intraveineuse (IgIV) et la plasmaphérèse (Shorer Z, 1997). Les corticoïdes en monothérapie sont contre indiqués (Hughes RA et al, 2006). Les soins de support (réanimation de base, nutrition, kinésithérapie) gardent une place importante (Thomas NH, 2005). D'une manière générale, il s'agit d'une affection de bon pronostic si la prise en charge est précoce et adéquate mais des séquelles invalidantes peuvent persister (Van Doorn PA, 2013). Nous rapportons deux observations d'enfants ayant présenté un syndrome de Guillain-Barré d'évolution favorable sous traitement symptomatique dans un contexte à ressources limitées. 


\section{Cas cliniques}

\section{Cas n ${ }^{\circ} 1$}

Il s'agit d'une fille de 12 ans, sans antécédents particuliers, ayant reçu tous les vaccins du programme élargi de vaccination (PEV), admise pour dysphagie, dysphonie, difficultés respiratoires, asthénie intense et impotence fonctionnelle des quatre membres d'aggravation progressive et d'évolution ascendante depuis deux jours. Un syndrome grippal fait de rhinorrhée antérieure claire, de céphalées avec asthénie associé à un paludisme a été rapporté deux semaines avant le début des symptômes. L'examen clinique à l'admission retrouvait une enfant asthénique, prostrée, apyrétique, avec une conscience normale et des signes évidents de déshydratation. L'examen des paires crâniennes retrouvait une atteinte des nerfs mixtes (IX, X et XI). Il n'y avait pas de paralysie faciale ni de troubles oculomoteurs. Les pupilles étaient de taille normale, symétriques et photoréactives. Les réflexes ostéotendineux et cutanés abdominaux étaient abolis. Un signe de Babinski bilatéral était présent. La sensibilité superficielle était conservée. La force motrice était estimée à $2 / 5$ aux quatre membres. Il y avait une tachypnée et des signes de lutte respiratoire. Le score de Silverman était évalué à 6/10. L'abdomen était sensible à la palpation dans la région périombilicale. L'examen ORL y compris la nasofibroscopie était normal. L'analyse du liquide céphalorachidien retrouvait une dissociation albumino-cytologique (cytologie zéro élément, proteinorachie $1,04 \mathrm{~g} / 1$ et glucorachie normale). Le bilan biologique notait une hyperleucocytose à prédominance neutrophile, une vitesse de sédimentation accélérée à $88 \mathrm{~mm}$ à la première heure ; la sérologie rétrovirale était négative. L'électromyographie (EMG) a permis de confirmer le diagnostic de SGB type axono-myelinique ; La prise en charge a consisté en une désobstruction rhinopharyngée régulière associée à une oxygénothérapie, une réhydratation, une renutrition par sonde nasogastrique et des soins de nursing. La kinésithérapie a été démarrée dès amélioration de l'état respiratoire. L'évolution a été marquée par une amélioration de l'état respiratoire et la récupération de la voix à $\mathrm{J} 7$. La station debout et la marche avec aide étaient possibles à J12 avec une FM 3/5 puis la marche autonome a été possible à J21 avec persistance d'une paraparésie. Avec un recul de 4 mois, l'examen neurologique était normal.

\section{$\operatorname{Cas} n^{\circ} 2$}

Il s'agit d'un nourrisson de 17 mois de sexe masculin, sans antécédents particuliers, bien vacciné selon le PEV en vigueur, admis pour difficultés respiratoires et altération de l'état général évoluant depuis trois jours sans autres signes associés. Pas de vaccination ni d'épisode infectieux récent rapporté. L'examen clinique à l'admission retrouvait une conscience normale, une irritabilité, une dysphonie avec des pleurs presque inaudibles, une détresse 
respiratoire sévère, une tachypnée avec signes de lutte respiratoires et un score de Silverman à 6/10; L'auscultation pulmonaire et cardiaque était normale. Il n’y avait pas de paralysie oculomotrice ni de paralysie faciale. On retrouvait une tétraparésie ; la force motrice était évaluée à $2 / 5$. Le nourrisson présentait une hypotonie axiale qui rendait la station assise impossible alors que le développement psychomoteur antérieur était normal. Il n'y avait pas de troubles sensitifs. Tous les réflexes ostéo-tendineux étaient abolis. Sur le plan paraclinique, l'étude du liquide céphalorachidien révélait une dissociation albumino-cytologique (cytologie=zéro élément, proteïnorachie $=1,85 \mathrm{~g} / 1$, glucorachie normale). La goutte épaisse, l'hémogramme et la $\mathrm{C}$ reactive protein étaient normaux; la sérologie rétrovirale était négative. L'électromyographie n'avait pas été réalisée. La prise en charge était symptomatique comme précédemment décrite. L'évolution a été marquée par une persistance de la dysphonie et de la détresse respiratoire jusqu'à $\mathrm{J} 8$; le début de récupération de la force motrice s'est faite à partir de J5 et la station assise a été possible au $12^{\text {ième }}$ jour. Le nourrisson est sorti de l'hôpital au $14^{\text {ième }}$ jour. Avec un recul de 4 mois, son examen neurologique est normal pour son âge.

\section{Commentaires}

Le SGB est rare en pédiatrie mais il représente la première cause de paralysie flasque aigue dans cette tranche d'âge depuis la mise en œuvre des protocoles d'éradication de la poliomyélite antérieure aigue (Halawa et al, 2011). La survenue de formes atypiques de diagnostic difficile n'est pas rare en pédiatrie (Pons L et al, 2015).

Nos deux patients ont été admis à deux semaines d'intervalle ; Il s'agit probablement d'une coïncidence car la majorité des cas rapportés surviennent sporadiquement. En moyenne 1,6 à 2,6 cas par an dans les études algérienne (Ahmane et al, 2014) et tunisienne (Sfaihi L et al, 2014). Certaines études ont retrouvé une prédominance saisonnière de l'affection comme en Chine (Wu X et al, 2016) et au Mexique (Nachamkin I et al, 2007) où elle serait plus fréquente en été et au printemps.

Nos patients étaient âgés respectivement de 12 ans et 17 mois. Les moyennes d'âge des différents auteurs se situent entre 6 et 10 ans. La survenue avant 2 ans est rare (Akbayram S et al, 2011) mais l'affection peut survenir chez le nouveau né comme rapporté par Anastopuolos et al en 2016. La prédominance masculine est constante dans toutes les études ; en moyenne les hommes sont 1,5 fois plus touchés que les femmes.

Notre patiente a présenté un syndrome grippal associé à un paludisme deux semaines avant le début des signes neurologiques alors que le nourrisson n'a présenté aucun symptôme avant le début de la maladie. Cette constatation concorde avec les données des différentes séries où on ne retrouve cet 
antécédent de maladie que dans un cas sur deux et ceci dans les 6 semaines précédentes. Les signes respiratoires et digestifs sont les plus fréquemment en cause.

Sur le plan clinique, nos deux patients présentaient une tétraparésie, une dysphonie, des difficultés respiratoires et une aréflexie tendineuse. Dans la littérature, le déficit moteur est présent dans 90 à 100\% des cas. L'atteinte des paires crâniennes est présente chez un patient sur quatre. Moins de $10 \%$ des enfants atteints de SGB nécessiteront une assistance ventilatoire (Salehiomran MR et al, 2016). La forme démyélinisante est la plus fréquente en pédiatrie et son pronostic est meilleur en comparaison aux formes axonales. Nos deux patients ont présenté une dissociation albuminocytologique à l'étude du liquide céphalorachidien. Cette donnée essentielle au diagnostic est très inconstamment retrouvée dans la littérature. La sérologie rétrovirale était négative chez nos deux patients même si le SGB peut être inaugural d'une infection à VIH (Conlon CP, 1989).

L'évolution naturelle du SGB peut être favorable comme dans le cas de nos deux patients mais l'hospitalisation est souvent plus longue de même que les délais de récupération en l'absence d'administration des Ig IV. En effet, nos patients ont été hospitalisés respectivement pendant 3 et 2 semaines alors que les moyennes dans la littérature tournent autour de 5 jours si les Ig IV sont administrées précocement avec un taux de récupération totale à $85 \%$ en 6 à 12 mois (Erazo Tonicelli R, 2009). Les taux de mortalité au cours du SGB chez l'enfant sont très faibles et varient dans les différentes études entre 0 et $8,3 \%$. Le taux de $16 \%$ enregistré au cours de l'étude égyptienne (Halawa et al, 2011) s'expliquait par une proportion élevée d'atteinte dysautonomique.

\section{Conclusion}

Le SGB est une affection de bon pronostic en pédiatrie si la prise en charge est rapide et correcte. Toutes les études recommandent une administration précoce des Ig IV dès la suspicion clinique de SGB de même qu'une prise en charge symptomatique adéquate pour avoir les meilleures chances de récupération complète car si le taux de mortalité est faible, des séquelles invalidantes peuvent handicaper l'enfant à vie.

CONFLIT D'INTERET : Aucun.

\section{References:}

1. AHMANE H, ARHAB D, BENSADI N. (2014). Syndrome de Guillain-Barré : Expérience d'un service de pédiatrie. Arch Ped ; 21:333-990. 
2. AKBAYRAM S, DOGAN M, AKGUN C, et al. (2011).Clinical features and prognosis with Guillain- Barré syndrome. Ann Indian Acad Neurol; 14(2):98-102.

3. ANASTASOPOULOU S, LINDEFELDT M, BARTOCCI M, WICKSTRÖM R. (2016). Acute inflammatory demyelinating polyradiculoneuropathy in a newborn infant. European journal of peadiatric neurology;20 :754-757.

4. CONLON CP.(1989). HIV infection presenting as Guillain-Barré syndrome in Lusaka, Zambia. Trans R Soc Trop Med Hyg; 83(1) :109.

5. ERAZO TONICELLI R. (2009). Guillain-Barré syndrome in pediatrics. Medecina ;69:84-91.

6. GUILLAIN G, BARRE JA, STROHL A.(1916). Sur un syndrome de radiculo-névrite avec hyperalbuminose du liquide céphalo-rachidien sans réaction cellulaire. Remarques sur les caractères cliniques et graphiques des réflexes tendineux. Bull Soc Méd hôp Paris; 40 :146270.

7. HALAWA EF, AHMED D, NADA MAF. (2011). Guillain-Barré syndrome as a prominent cause of childhood acute flaccid paralysis in post polio eradication era in Egypt. European journal of peadiatric neurology; 15 :241-246.

8. HUGHES R, CORNBLATH D. (2005). Guillain- Barré syndrome. Lancet; 366 :1653-66.

9. HUGHES RA, SWAN AV, VAN KONINGSVELD R, VAN DOORN PA. (2006). Corticosteroids for Guillain-Barré syndrome. Cochrane Database Syst Rev; 2 :CD001446.

10. KUWABARA S. (2004). Guillain-Barré syndrome : epidemiology, pathophysiology and management. Drugs; 64(6) :597-610.

11. NACHAMKIN I, ARZATE BARBOSA P, UNG H, et al. (2007). Pattern of Guillain-Barré syndrome in children: results from a mexican population. Neurology; 69(17) :1665-71.

12. PONS L, MANEL V, VILLE D, JAVOUHEY E, BORDET F.(2015). A new observation of an atypical and severe variant of Guillain-Barré syndrome in a child : remaining challenges for diagnosis, nosologic classification, and therapeutic course. Child Neurology Open :1-6. 
13. SALEHIOMRAN MR, NIKKAH A, MAHDAVI M. (2016). Prognosis of Guillain- Barré syndrome in children. Iran J Child Neurol. Spring ;10(2):38-41.

14. SFAIHI L, TALMOUDI J,MAALOUL I, KAMOUN F, KMIHA S, BEN AMEUR S, KAMOUN T, HACHICHA M. (2014). Polyradiculonévrite aigüe primitive de l'enfant : à propos de 20 cas. Arch Ped ; 21 :333-990.

15. SHORER Z. (1997). Current treatment in acute demyelinating polyneuropathy (Guillain-Barré syndrome). European journal of peadiatric neurology; $1: 3-5$.

16. THOMAS NH.(2005). Diagnosis and management of Guillain-Barré syndrome. Current Paediatrics ; 15:287-291.

17. VAN DOORN PA. (2013). Diagnosis, treatment and prognosis of Guillain-Barré syndrome. Presse Med.;42(6)

18. WU X, SHEN D, LI T. (2016). Distinct clinical characteristics of pediatric Guillain-barré syndrome: a comparative study between children and adults in north east China. PLOS ONE/ DOI : 10.1371 /journal.pone0151611. 